\title{
Vehicle Sideslip Angle Estimation Based on General Regression Neural Network
}

\author{
Wang Wei, Bei Shaoyi, Zhang Lanchun, Zhu Kai, Wang Yongzhi, and Hang Weixing \\ School of Automotive and Traffic Engineering, Jiangsu University of Technology, Jiangsu, Changzhou 213001, China \\ Correspondence should be addressed to Bei Shaoyi; beishaoyi@126.com
}

Received 15 September 2015; Revised 17 March 2016; Accepted 24 March 2016

Academic Editor: Fei Liu

Copyright (C) 2016 Wang Wei et al. This is an open access article distributed under the Creative Commons Attribution License, which permits unrestricted use, distribution, and reproduction in any medium, provided the original work is properly cited.

\begin{abstract}
Aiming at the accuracy of estimation of vehicle's mass center sideslip angle, an estimation method of slip angle based on general regression neural network (GRNN) and driver-vehicle closed-loop system has been proposed: regarding vehicle's sideslip angle as time series mapping of yaw speed and lateral acceleration; using homogeneous design project to optimize the training samples; building the mapping relationship among sideslip angle, yaw speed, and lateral acceleration; at the same time, using experimental method to measure vehicle's sideslip angle to verify validity of this method. Estimation results of neural network and real vehicle experiment show the same changing tendency. The mean of error is within $10 \%$ of test result's amplitude. Results show GRNN can estimate vehicle's sideslip angle correctly. It can offer a reference to the application of vehicle's stability control system on vehicle's state estimation.
\end{abstract}

\section{Introduction}

Electronic stability program (ESP) which includes antilock brake system (ABS), traction control system (TCS), and active yaw control system (AYC) can improve vehicle's maneuverability in the extreme driving situation. The improvement of vehicle's control ability depends on the accuracy estimation of vehicle's kinematics states. Vehicle's mass center sideslip angle is an important index of vehicle's stability. It is difficult to observe sideslip angle preciously and timely according to ESP sensor signals $[1,2]$. Now, the common methods include fuzzy logic estimation method [3], Kalman filter method $[4,5]$, state observer method (based on lateral speed observation) [6], and synthetic method (combination of integration method and state observation) $[7,8]$. The above methods are mainly based on the vehicle model. The observation accuracy and real-time depend on the complexity of model.

The neural network technology is used to estimate vehicle's sideslip angle in this paper. Yaw angle acceleration and lateral acceleration are used to estimate vehicle body's sideslip angle based on back propagation (BP) neural network in the literature [9], and the satisfactory result is attained. However,
BP neural network has disadvantages of slowly convergence speed and local minimum value. But radial basis function (RBF) neural network is a kind of feed forward neural network with high performance. It needs less calculation and has better efficient learning speed. It also has a stronger ability of parameter approximation and classification than BP neural network. GRNN is a special form of radial basis function neural network $[10,11]$. Its abilities of approximate function and learning are very strong. Thus this special radial basis function neural network could be used in this paper to estimate the vehicle sideslip angle.

\section{Driver-Vehicle Closed-Loop Model}

2.1. Vehicle Model. In the model, the vehicle travels at a constant speed $u$; the inertia and damping of the steering system, the effect of suspension, the cornering property change of left and right tires due to different vertical loads, and the influence of tire aligning torque are all ignored. As shown in Figure 1, two generalized coordinates, yawing angle $\psi$ and centroid sideslip angle $\beta$, are utilized to represent the vehicle's motion state. 


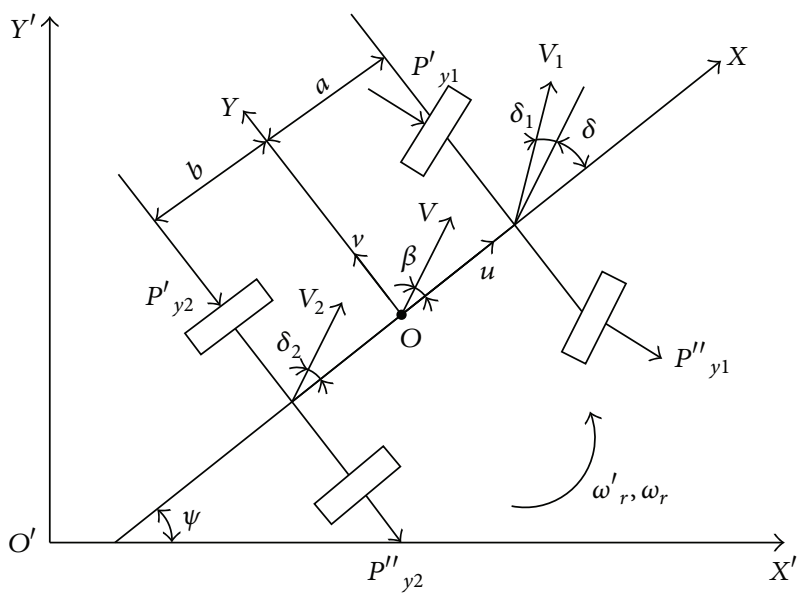

FIgURE 1: 2DOF vehicle model.

According to D'Alembert principle, the force equilibrium equations of the vehicle's motion are as follows:

$$
\begin{aligned}
M u\left(\omega_{r}+\dot{\beta}\right) & =-\left(P_{y 1}+P_{y 2}\right), \\
I_{Z} \dot{\omega}_{r} & =-a P_{y 1}+b P_{y 2},
\end{aligned}
$$

where $I_{Z}$ is rotational inertia of the vehicle around $z$-axis and $P_{y 1}, P_{y 2}$ are cornering force of front and rear wheels, respectively.

2.2. Driver Model. According to the "preview optimal curvature control theory," the driver-vehicle system block can be presented as shown in Figure 2, where $f(s)$ is the road input, $y(s)$ is the lateral displacement response, $V(s)$ is the vehicle's transfer function, $T$ is the driver's preview time, $T_{C}$ is the correction time, $T_{d}$ is the neural lag time, $T_{h}$ is the operation lag time, $C_{0}$ is the correction parameter, $\ddot{y}^{*}$ is the ideal lateral acceleration, $\delta_{\mathrm{sw}}^{*}$ is the ideal steering-wheel angle, and $\delta_{\mathrm{sw}}$ is actual steering-wheel angle.

2.3. Closed-Loop Model. The state equation of driver-vehicle 2 DOF closed-loop model will be obtained after vehicle's model and driver model are confirmed:

$$
\left[\begin{array}{c}
\dot{\omega}_{r} \\
\dot{\beta} \\
\dot{y} \\
\dot{v}_{a} \\
\dot{x}_{1} \\
\dot{x}_{2}
\end{array}\right]=\left[\begin{array}{cccccc}
a_{11} & a_{12} & 0 & 0 & a_{15} & a_{16} \\
a_{21} & a_{22} & 0 & 0 & a_{25} & a_{26} \\
0 & 0 & 0 & 1 & 0 & 0 \\
a_{41} & a_{42} & 0 & 0 & a_{45} & a_{46} \\
0 & 0 & 0 & 0 & 0 & 1 \\
a_{61} & a_{62} & a_{63} & a_{64} & a_{65} & a_{66}
\end{array}\right]\left[\begin{array}{c}
\omega_{r} \\
\beta \\
y \\
v \\
x_{1} \\
x_{2}
\end{array}\right]
$$

$$
+\left[\begin{array}{l}
0 \\
0 \\
0 \\
0 \\
0 \\
b_{6}
\end{array}\right] f_{r}
$$

The output equation is as follows:

$$
\begin{aligned}
{\left[\begin{array}{l}
y \\
a_{y} \\
\delta_{\mathrm{sw}} \\
\dot{\delta}_{\mathrm{sw}} \\
S_{1} \\
S_{2}
\end{array}\right]=} & {\left[\begin{array}{cccccc}
0 & 0 & c_{13} & 0 & 0 & 0 \\
c_{21} & c_{22} & 0 & 0 & c_{25} & c_{26} \\
0 & 0 & 0 & 0 & c_{35} & c_{36} \\
c_{41} & c_{42} & c_{43} & c_{44} & c_{45} & c_{46} \\
c_{51} & c_{52} & 0 & 0 & c_{55} & c_{56} \\
c_{61} & c_{62} & 0 & 0 & 0 & 0
\end{array}\right]\left[\begin{array}{c}
r \\
\beta \\
y \\
v_{a} \\
x_{1} \\
x_{2}
\end{array}\right] } \\
& +\left[\begin{array}{c}
0 \\
0 \\
0 \\
d_{4} \\
0 \\
0
\end{array}\right] f_{r},
\end{aligned}
$$

where $f_{r}=f(t+T)+T_{c} \dot{f}(t+T), r$ is vehicle's yaw angle acceleration, $\beta$ is sideslip angle of mass center, $v_{a}$ is vehicle's lateral acceleration, $S_{1}$ and $S_{2}$ are lateral force coefficient of front and back wheel, respectively, and $x_{1}, x_{2}$ are state variables of vehicle's model.

\section{Road Model}

Double lane and serpentine lines are typical road models that are commonly used in vehicle handling stability evaluation. To simulate vehicle state estimation, double lane and serpentine lines are used as the ideal road input. Meanwhile, the road trace takes the actual road centerline trajectory.

3.1. Double Lane Road Input Model. Double lane road test model dimensions are shown in Figure 3. The actual road segment size parameters in Figure 4 are $s_{0}=s_{1}=s_{2}=$ $s_{4}=2 u, s_{3}=u, s_{5}=5 u$, and $s_{6}=3 u$, where $u$ is the car's driving speed, and the changing lanes distance width $B=$ $3.5 \mathrm{~m}$ (assumed to be a standard motor vehicle lane width).

Take the centerline of the test road for the ideal way trajectory to track in the driver mind. Because the way in the corner points has a mutation, the car's actual travel path cannot have a mutation, so smooth handling is conducted in the mutation department to make the road close to the ideal trajectory. The most simple and effective treatment is carrying out third-order curve to fit to this polyline, so after fitting the road functions and a derivative at break point are continuous. 


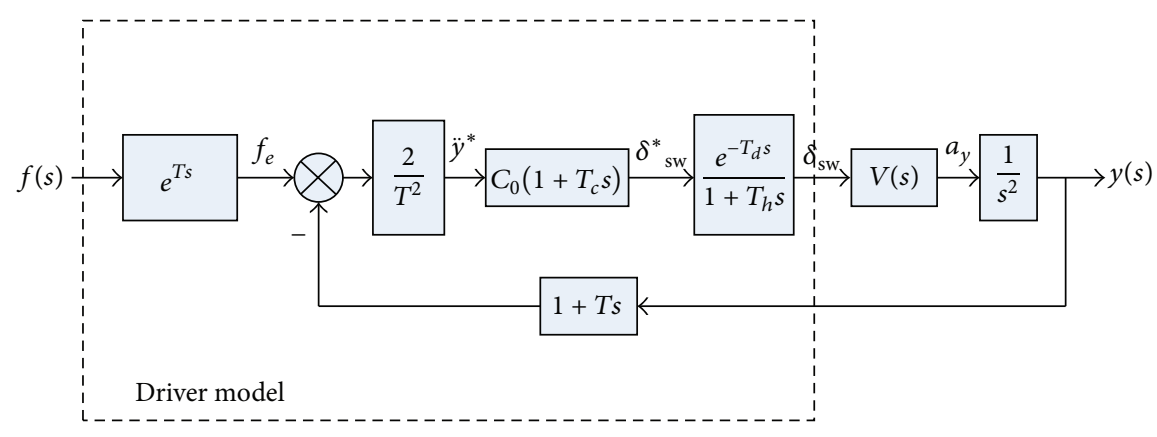

FIgURE 2: Driver-vehicle closed-loop system.

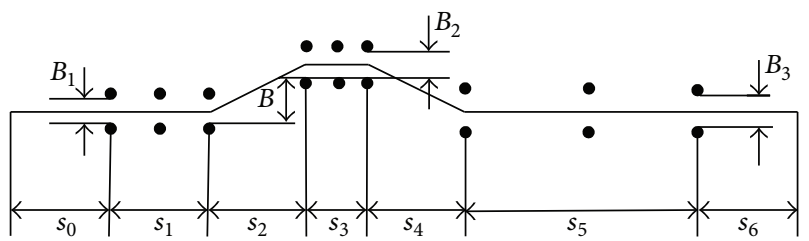

FIgURE 3: Double lane road test model diagram.

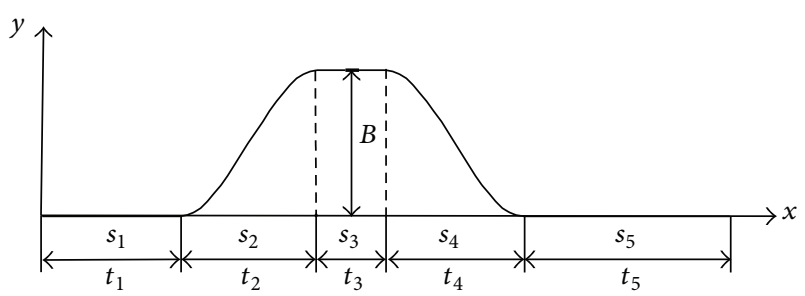

FIgURE 4: A double shift line graphafter the third-order curve fit.

The ideal path input after third-order curve fit is shown in Figure 4.

The abscissa $x$ is the longitudinal displacement of the car, and the ordinate $y$ is the lateral displacement of the car.

The third-order curve expression after fitting is

$$
f(x)= \begin{cases}0 & x \in s_{1} \\ e_{0}+e_{1} x+e_{2} x^{2}+e_{3} x^{3} & x \in s_{2} \\ B & x \in s_{3} \\ e_{0}^{\prime}+e_{1}^{\prime} x+e_{2}^{\prime} x^{2}+e_{3}^{\prime} x^{3} & x \in s_{4} \\ 0 & x \in s_{5},\end{cases}
$$

where parameters are as follows:

$$
\begin{aligned}
& a_{0}=s_{1}, \\
& a_{1}=s_{1}+s_{2}, \\
& a_{2}=s_{1}+s_{2}+s_{3}, \\
& a_{3}=s_{1}+s_{2}+s_{3}+s_{4}, \\
& d=a_{1}-a_{0}, \\
& d^{\prime}=a_{2}-a_{3},
\end{aligned}
$$

$$
\begin{aligned}
& e_{0}=\frac{a_{0}^{2}\left(3 a_{1}-a_{0}\right) B}{d^{3}}, \\
& e_{1}=-\frac{6 a_{0} a_{1} B}{d^{3}}, \\
& e_{2}=\frac{3\left(a_{0}+a_{1}\right) B}{d^{3}}, \\
& e_{3}=-\frac{2 B}{d^{3}}, \\
& e_{0}^{\prime}=\frac{a_{3}^{2}\left(3 a_{2}-a_{3}\right) B}{d^{\prime 3}}, \\
& e_{1}^{\prime}=-\frac{6 a_{3} a_{2} B}{d^{\prime 3}}, \\
& e_{2}^{\prime}=\frac{3\left(a_{3}+a_{2}\right) B}{d^{\prime 3}}, \\
& e_{3}^{\prime}=-\frac{2 B}{d^{\prime 3}} .
\end{aligned}
$$

Longitudinal displacement of the car can be obtained by $x=u t$, and the function $f(x)$ in formula (4) can be converted into a function of time $f(t)$. The specific expressions are

$$
f(t)= \begin{cases}0 & t \in t_{1} \\ g_{0}+g_{1} t+g_{2} t^{2}+g_{3} t^{3} & t \in t_{2} \\ b & t \in t_{3} \\ h_{0}+h_{1} t+h_{2} t^{2}+h_{3} t^{3} & t \in t_{4} \\ 0 & t \in t_{5},\end{cases}
$$

where $g_{0}=e_{0}, g_{i}=e_{i} u^{j}(j=1,2,3), h_{0}=e_{0}^{\prime}$, and $h_{i}=$ $e_{i}^{\prime} u^{j}(j=1,2,3)$.

3.2. Serpentine Road Input Model. Serpentine test road model dimensions are shown in Figure 5.

The actual road segment size parameters in Figure 5 are $s_{0}=L=2 u, 5 L=10 u$, and $s=3 u$. $u$ is vehicle speed; pole width $B=2.46 \mathrm{~m}$ (the width of the text is designed according to the car models in the test). 


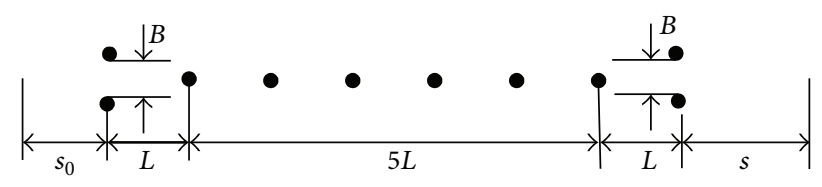

FIGURE 5: Serpentine road input model diagram.

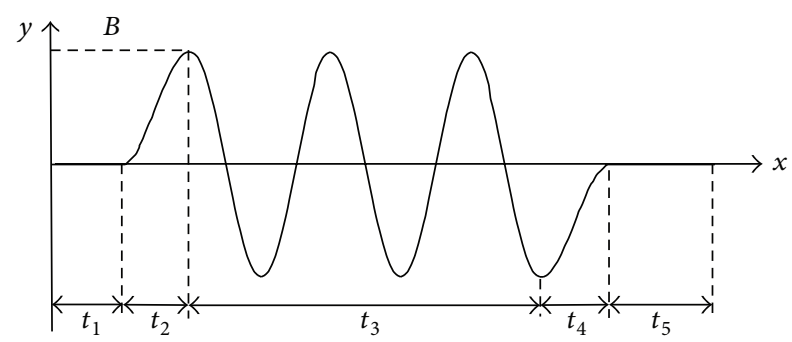

Figure 6: A serpentine line graph after the third-order curve fit.

The ideal tracking trajectory by an actual driver will be shown in Figure 6. After a cubic fitting the road, the thirdorder curve with continuous derivative is as follows.

The serpentine road function of time after fitting is as follows (a similar derivation with double lane, so we will not repeat them but only give results):

$$
f(t)= \begin{cases}0 & t \in t_{1} \\ \frac{H(1.0+\sin (\pi(t+1) / 2))}{2.0} & t \in t_{2} \\ H \cos \left(\frac{\pi t}{2}\right) & t \in t_{3} \\ \frac{H(-1.0+\sin (\pi(t+1) / 2))}{2.0} & t \in t_{4} \\ 0 & t \in t_{5},\end{cases}
$$

where $H=B / 2$.

\section{Generalized Regression Neural Network Structure}

Generalized regression neural network (GRNN) is a special form of the RBF neural network. GRNN has a good performance in function approximation and learning ability. So it can be used in this paper. Its topological structure is shown in Figure 7.

The neurons of first layer in GRNN have the same function of the RBF. The difference between GRNN and RBF is the special linear layer. The output of radial basis layer in GRNN computes with weight matrices LW $_{2,1}$ by using "nprod" calculation method which does not compute in RBF. Then the result is sent to linear transfer function. The above process could be accomplished with neural network toolbox in MATLAB.

The number of radial basis and special linear neurons in GRNN is the same as the input sample vector. The weight matrix $\mathrm{LW}_{2,1}$ is set as output vector [T]. So, if the input of real network is 1 , the objective output is much closer to the network output vector $[T]$. Therefore, the ability of function approximation of GRNN is better than basic radial basis neural network in general problems.

\section{Sideslip Angle Neural Network Estimation Model}

The neural network model of vehicle sideslip angle is based on the following assumption. The vehicle sideslip angle can be expressed as function of yaw rate and lateral acceleration [12]:

$$
\begin{aligned}
& \beta(k+1)=f\left(\omega_{r}(k), \omega_{r}(k-1), \ldots, \omega_{r}(k-n), a_{y}(k),\right. \\
& \left.\quad a_{y}(k-1), \ldots, a_{y}(k-n)\right) .
\end{aligned}
$$

Sideslip angle $\beta$ of mass center at current $k+1$ time is the time series function which is composed of the yaw rate $\omega_{r}$ and lateral acceleration $a_{y}$ in previous $n+1$ moments. Then their mapping relationship is established by using neural networks. The $n$ decides the scale of neural networks. The study finds that $n=4$ will be suitable by considering both computing time and estimation precision.

Nervous response lag time $T_{d}$, control response lag time $T_{h}$, and preview time $T$ are parameters of describing driver's proficiency degree. Due to the differences among drivers, $T_{d}, T_{h}$, and $T$ have ranges $\left[T_{d}, \overline{T_{d}}\right],\left[T_{h}, \overline{T_{h}}\right]$, and $[\underline{T}, \bar{T}]$. By combining different values of three parameters within their ranges of variation to simulate different drivers driving the same car, multisamples are obtained to train networks.

In the theory, as long as training samples are enough and representative, neural networks can reveal the arbitrarily complex law which is contained in it. The efficient uniform design method is used to select the training samplesin this paper. In this way, not only does the number of training samples significantly reduces, but also each training sample is more representative. The uniform design table $U_{15}\left(15^{8}\right)$ which is 8 factors, 15 standards, and 15 times experiments is selected to simulate experiment design (as shown in Tables 1 and 2).

\section{Comparison between Estimates and Experimental Values}

The real vehicle tests of double lane and serpentine line have been done. Gyroscope is used to collect vehicle's yaw rate and lateral acceleration in real time. Contactless speed sensor is used to obtain vehicle's sideslip angle. The test road is set according to double lane and serpentine line test procedures ISO/3888 technical reports. Experienced driver drives the tested vehicle to simulate driver parameters. Speed of double lane test is $u=80 \mathrm{~km} / \mathrm{h}$ and serpentine line is $u=65 \mathrm{~km} / \mathrm{h}$. During the test period vehicle cannot touch benchmarks.

6.1. Double Lane. The measured data of yaw rate and lateral acceleration during double lane vehicle test are shown in Figures 8 and 9. The measured data in Figures 8 and 9 are input to the trained GRNN sideslip angle estimation model. 


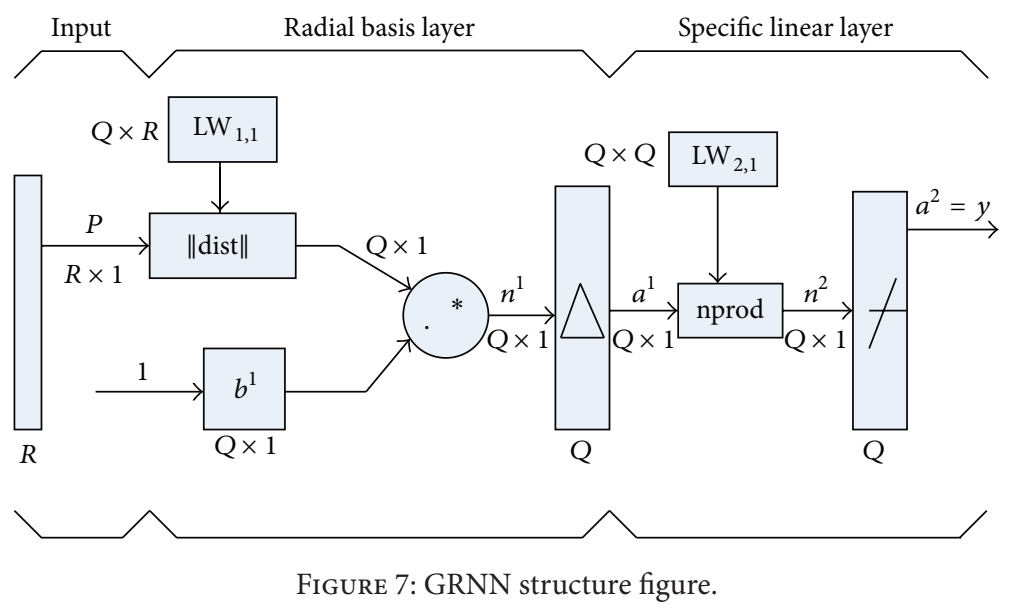

TABLE 1: $U_{15}\left(15^{8}\right)$ uniform design table.

\begin{tabular}{lcccccccc}
\hline \multirow{2}{*}{ Test } & \multicolumn{7}{c}{ Column } & \\
& 1 & 2 & 3 & 4 & 5 & 6 & 7 & 8 \\
\hline 1 & 1 & 2 & 4 & 7 & 8 & 11 & 12 & 14 \\
2 & 2 & 4 & 8 & 14 & 1 & 7 & 11 & 13 \\
3 & 3 & 6 & 12 & 6 & 9 & 3 & 9 & 12 \\
4 & 4 & 8 & 1 & 13 & 2 & 14 & 7 & 11 \\
5 & 5 & 10 & 5 & 5 & 10 & 10 & 5 & 10 \\
6 & 6 & 12 & 9 & 12 & 3 & 6 & 3 & 9 \\
7 & 7 & 14 & 13 & 4 & 11 & 2 & 1 & 8 \\
8 & 8 & 1 & 2 & 11 & 4 & 13 & 14 & 7 \\
9 & 9 & 3 & 6 & 3 & 12 & 9 & 12 & 6 \\
10 & 10 & 5 & 10 & 10 & 5 & 5 & 10 & 5 \\
11 & 11 & 7 & 14 & 2 & 13 & 1 & 8 & 4 \\
12 & 12 & 9 & 3 & 9 & 6 & 12 & 6 & 3 \\
13 & 13 & 11 & 7 & 1 & 14 & 8 & 4 & 2 \\
14 & 14 & 13 & 11 & 8 & 7 & 4 & 2 & 1 \\
15 & 15 & 15 & 15 & 15 & 15 & 15 & 15 & 15 \\
\hline
\end{tabular}

TABLE 2: $U_{15}\left(15^{8}\right)$ use table.

\begin{tabular}{|c|c|c|c|c|c|c|c|c|}
\hline \multirow{2}{*}{$\frac{\text { Factors }}{2}$} & \multicolumn{8}{|c|}{ Number of columns } \\
\hline & 1 & 6 & & & & & & \\
\hline 3 & 1 & 3 & 4 & & & & & \\
\hline 4 & 1 & 3 & 4 & 7 & & & & \\
\hline 5 & 1 & 2 & 3 & 4 & 7 & & & \\
\hline 6 & 1 & 2 & 3 & 4 & 6 & 8 & & \\
\hline 7 & 1 & 2 & 3 & 4 & 6 & 7 & 8 & \\
\hline 8 & 1 & 2 & 3 & 4 & 6 & 6 & 7 & 8 \\
\hline
\end{tabular}

Vehicle sideslip angle estimation curve $\beta_{\text {net }}$ is obtained. $\beta_{\text {real }}$ is obtained by experiment in Figure 10.

The trend of estimation values and test values match well from Figure 10. The error is under an allowable range of engineering application. The absolute error between $\beta_{\text {net }}$ and $\beta_{\text {real }}$ is small form Figure 11.

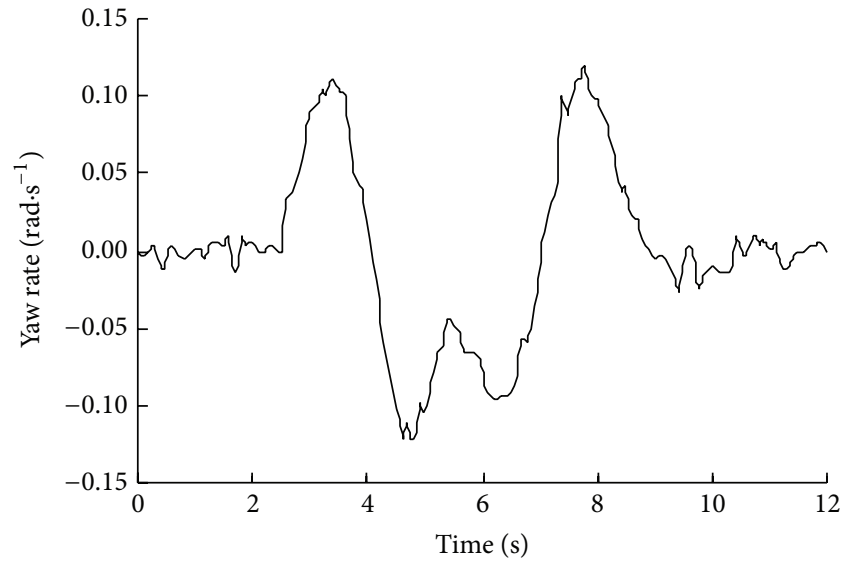

FIgURE 8: Vehicle yaw rate (double lane).

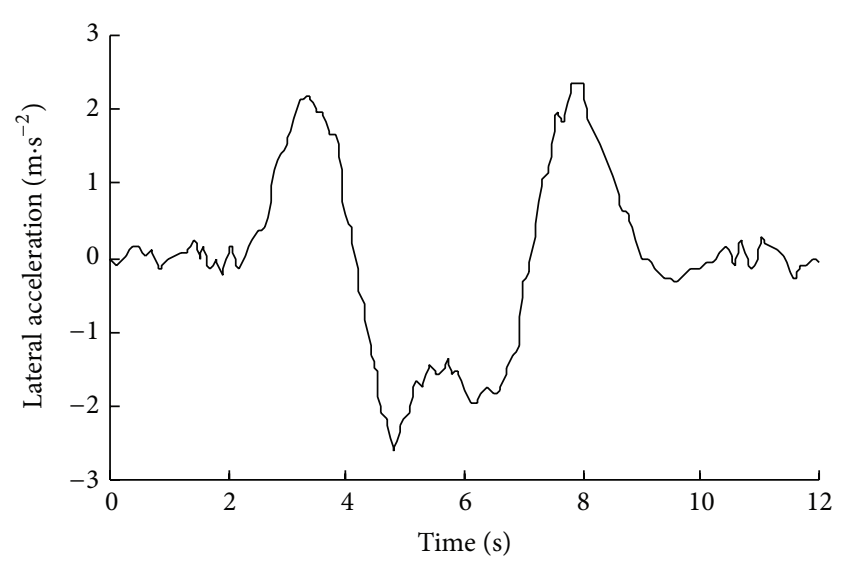

FIGURE 9: Vehicle lateral acceleration (double lane).

6.2. Serpentine Line. The measured data of yaw rate and lateral acceleration during serpentine line vehicle test are shown in Figures 12 and 13.

The measured data in Figures 12 and 13 is input to the trained GRNN sideslip angle estimation model. Vehicle sideslip angle estimation curve $\beta_{\text {net }}$ is obtained. $\beta_{\text {real }}$ is 


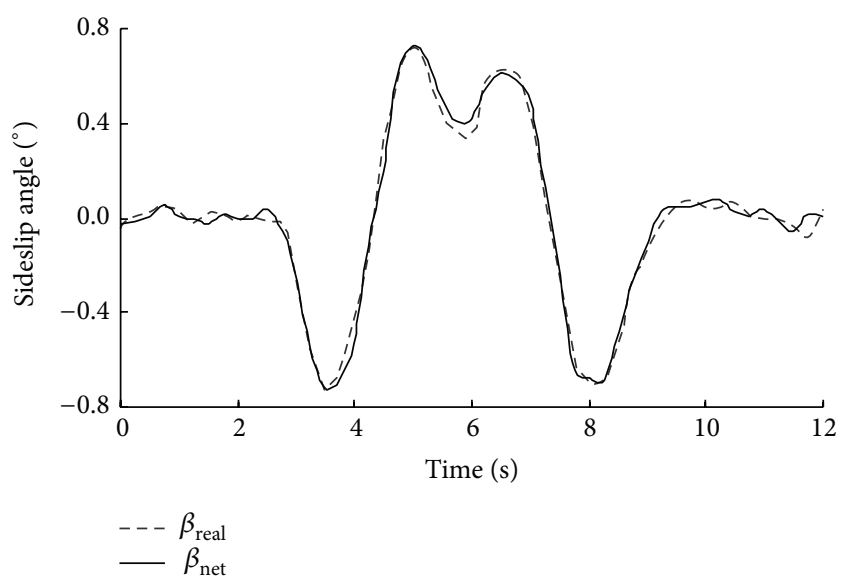

Figure 10: Comparison result (double lane).

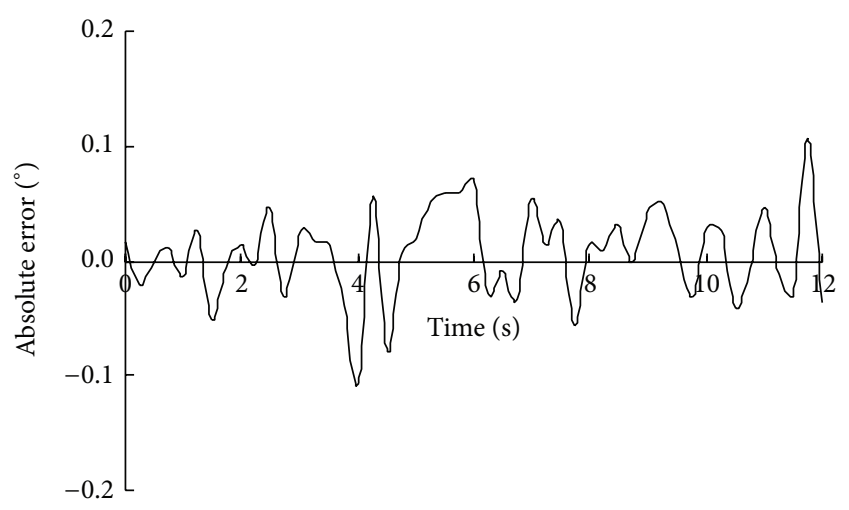

FIgURE 11: Absolute error (double lane).

TABLE 3: Error of neural network side angle estimation method.

\begin{tabular}{lcc}
\hline Error indicator & Double lane & Serpentine line \\
\hline Error mean & $0.029453^{\circ}$ & $0.037653^{\circ}$ \\
Error standard deviation & $0.042356^{\circ}$ & $0.049782^{\circ}$ \\
\hline
\end{tabular}

obtained by experiment in Figure 14, but estimation error of sideslip angle is larger in the relatively complicated serpentine line test from Figure 15.

In order to compare the two estimation methods quantitatively, absolute error of sideslip angle of neural networks estimation method and test method is given in Table 3.

Therefore, the vehicle sideslip angle estimation using GRNN method is simple, clear, and of high precision. The uniform design method greatly reduces the number of simulation, so the response time of the method is faster as well.

\section{Conclusions}

The estimation method of mass center sideslip angle based on GRNN and drive-vehicle closed-loop system are proposed in this paper. Vehicle sideslip angle is seen as the mapping of time series which is easily measured variables between yaw rate and lateral acceleration. The training samples are

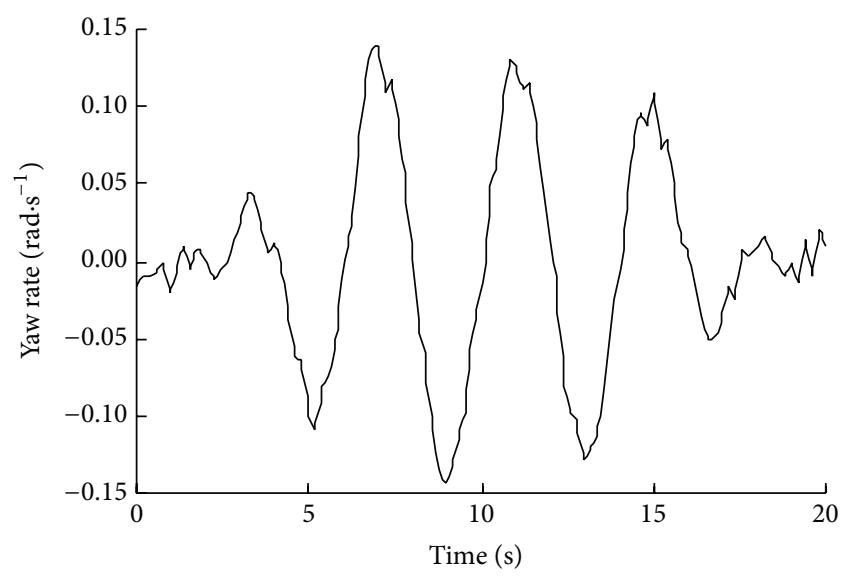

Figure 12: Vehicle yaw rate (serpentine line).

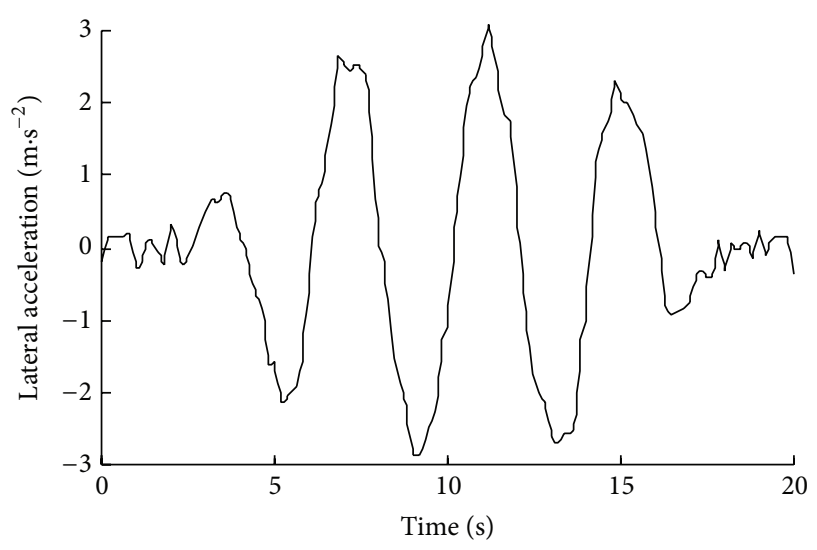

FIGURE 13: Vehicle lateral acceleration (serpentine line).

provided by using the efficient uniform design method. The trained GRNN has a high precision and fast response to estimate vehicle sideslip angle. The results show that GRNN has a high estimation precision and fast speed by comparing the estimation with experimental values. The main conclusions are as follows:

(1) In the vehicle side angle estimation test environment, the estimation speed of neural networks is close to real vehicle test data.

(2) Whether it is double lane or serpentine line condition, the estimation accuracy is high and the mean error is within $10 \%$ of the test amplitude.

(3) Due to the relatively high path complexity degree, the estimation error of serpentine line is bigger than double lane.

(4) Because of the high estimation accuracy and fast response speed, the neural network sideslip angle estimation method has a certain theoretical significance for vehicle dynamics control system design. It also can reduce vehicle's sideslip angle test cost and achieve the purpose of measuring flexibility in engineering application domain. 


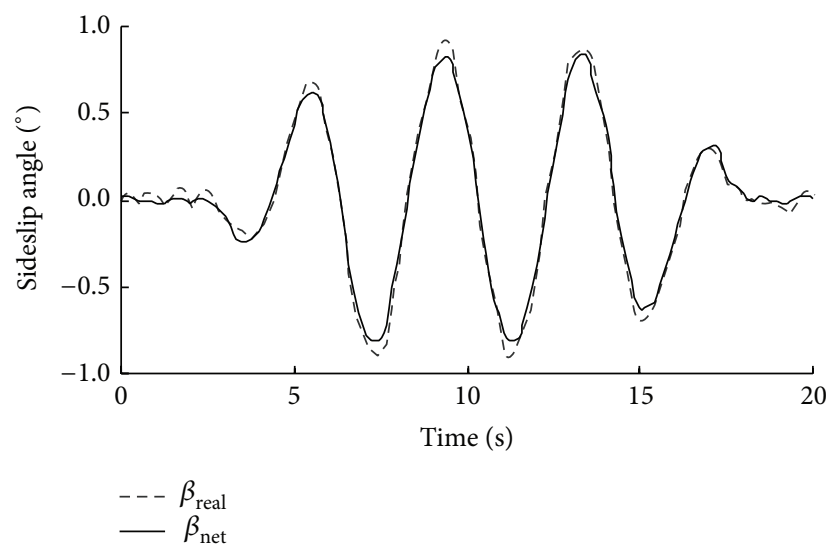

FIGURE 14: Comparison result (serpentine line).

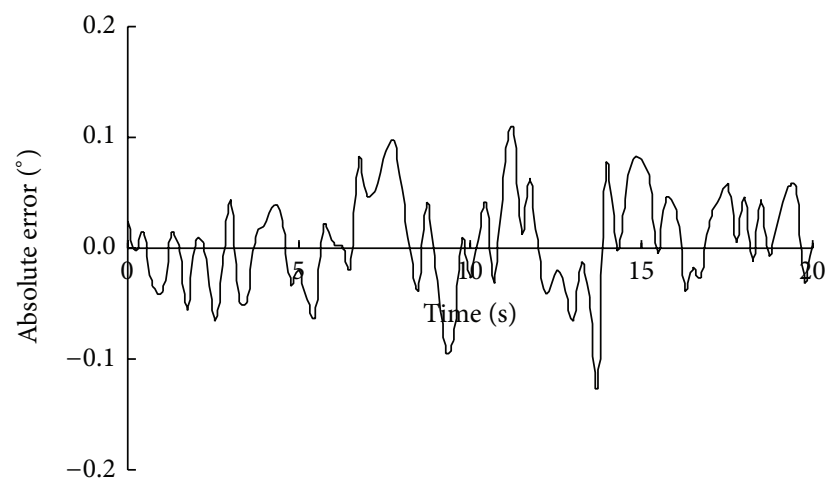

Figure 15: Absolute error (serpentine line).

\section{Competing Interests}

The authors declare that they have no competing interests.

\section{Acknowledgments}

This work is partially supported by the National Science Foundation of China (Grant no. 51305175), the National Science Foundation of Jiangsu Province (Grant no. BK2012586), the National Science Foundation of Jiangsu University of Technology (Grant no. KYY14041), the 333 Project of Jiangsu Province (BRA2015365), and Jiangsu province "six personnel peak" fund projects (Grant no. 2012-ZBZZ-023 and Grant no. 2013-ZBZZ-039). The authors also gratefully acknowledge the helpful comments and suggestions of the reviewers, which have improved the presentation.

\section{References}

[1] A. van Zanten, "Bosch ESP systems: 5 years of experience," SAE Technical Paper 2000-01-1633, SAE International, 2000.

[2] S.-H. You, J.-O. Hahn, and H. Lee, "New adaptive approaches to real-time estimation of vehicle sideslip angle," Control Engineering Practice, vol. 17, no. 12, pp. 1367-1379, 2009.

[3] S. Shuming, H. Lupker, and P. Bremmer, "Estimation of vehicle side slip angle based on fuzzy logic," Automotive Engineering, vol. 27, no. 4, pp. 426-430, 2005.
[4] P. J. T. Venhovens and K. Naab, "Vehicle dynamics estimation using Kalman filters," Vehicle System Dynamics, vol. 32, no. 2, pp. 171-184, 1999.

[5] B.-C. Chen and F.-C. Hsieh, "Sideslip angle estimation using extended Kalman filter," Vehicle System Dynamics, vol. 46, no. 1, pp. 353-364, 2008.

[6] L. Li, J. Song, L. Kong, and Q. Huang, "Vehicle velocity estimation for real-time dynamic stability control," International Journal of Automotive Technology, vol. 10, no. 6, pp. 675-685, 2009.

[7] Y. Fukada, "Slip-angle estimation for vehicle stability control," Vehicle System Dynamics, vol. 32, no. 4, pp. 375-388, 1999.

[8] W. J. Manning and D. A. Crolla, "A review of yaw rate and sideslip controllers for passenger vehicles," Transactions of the Institute of Measurement and Control, vol. 29, no. 2, pp. 117-135, 2007.

[9] H. Sasaki and T. Nishimaki, "A side-slip angle estimation using neural network for a wheeled vehicle," SAE 2000-01-0695, 2000.

[10] A. M. A. Haidar, M. W. Mustafa, F. A. F. Ibrahim, and I. A. Ahmed, "Transient stability evaluation of electrical power system using generalized regression neural networks," Applied Soft Computing, vol. 11, no. 4, pp. 3558-3570, 2011.

[11] I. J. Su, C. C. Tsai, and W. T. Sung, "Comparison of BP and GRNN algorithm for factory monitoring," Applied Mechanics and Materials, vol. 52-54, pp. 2105-2110, 2011.

[12] S. Hideaki and N. Takatoshi, "A side-slip angle estimation using neural network for a wheeled vehicle," SAE Technical Paper 2000-01-0695, SAE International, 2000. 


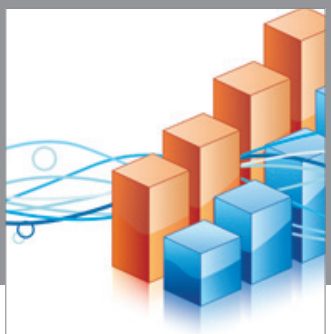

Advances in

Operations Research

vatem alat4

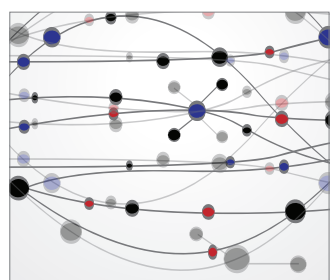

\section{The Scientific} World Journal
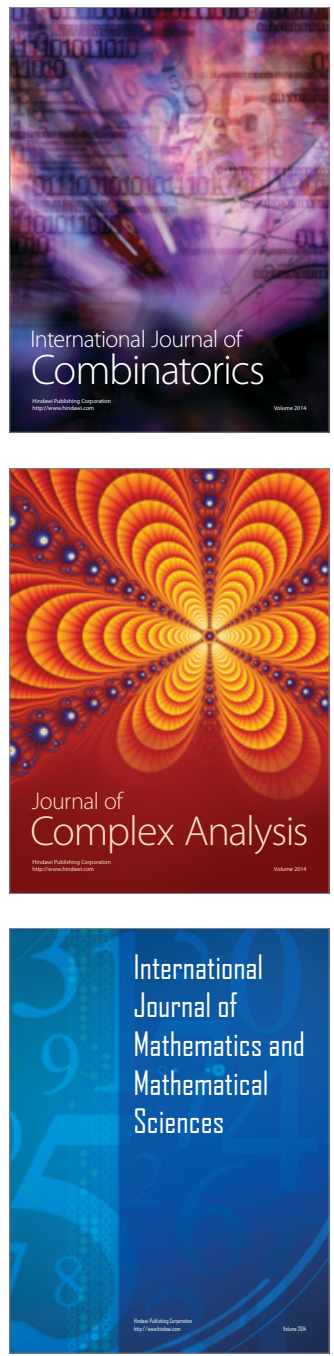
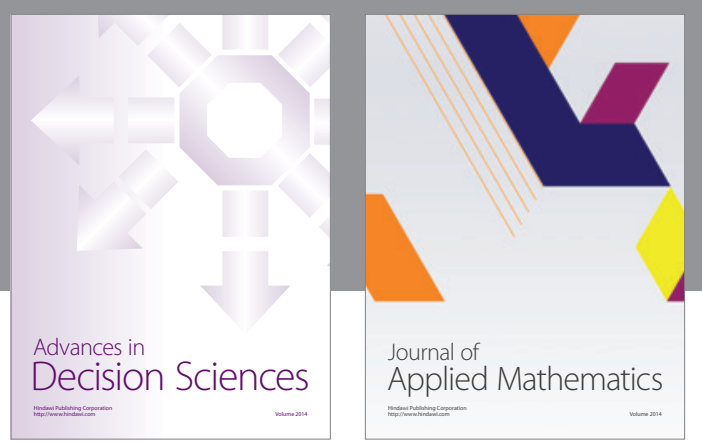

Algebra

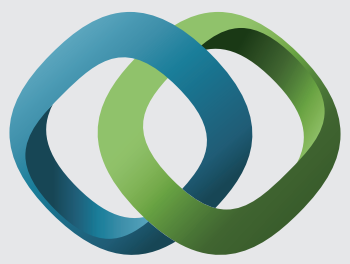

\section{Hindawi}

Submit your manuscripts at

http://www.hindawi.com
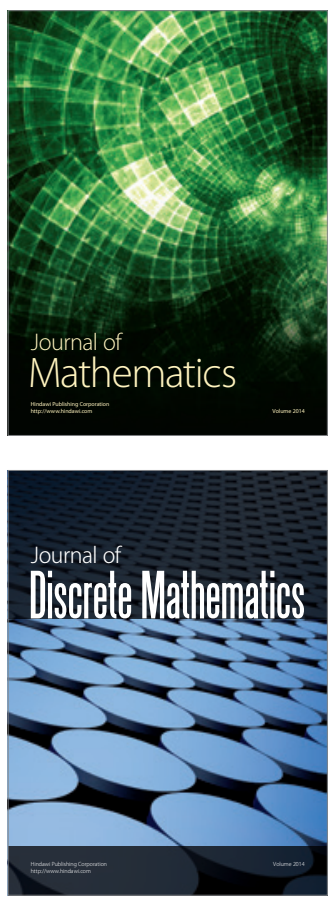

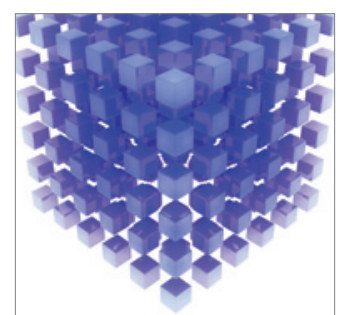

Mathematical Problems in Engineering
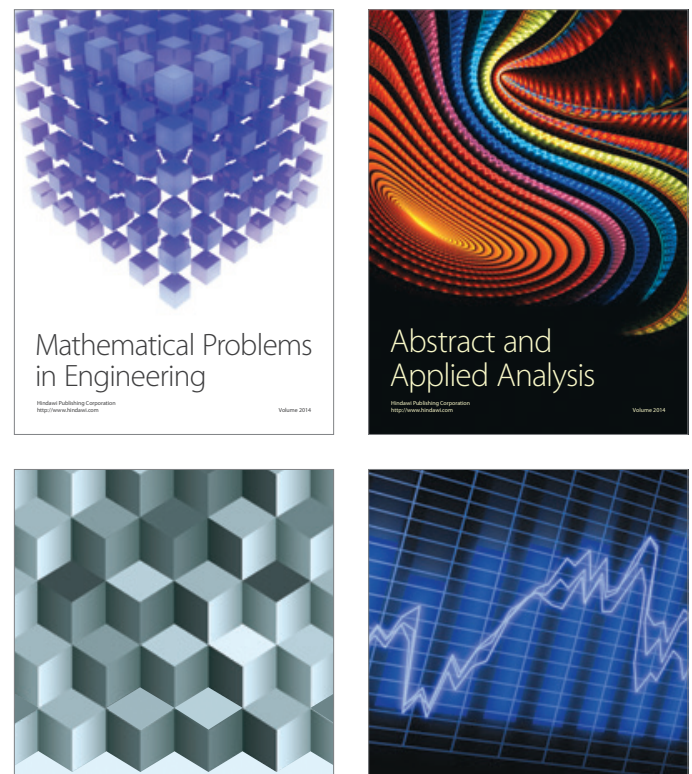

Journal of

Function Spaces

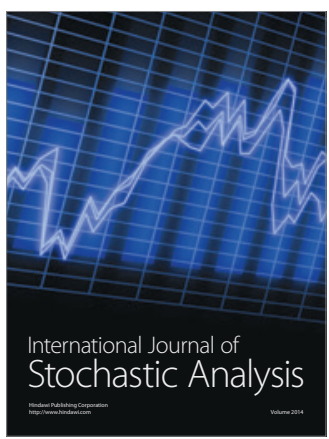

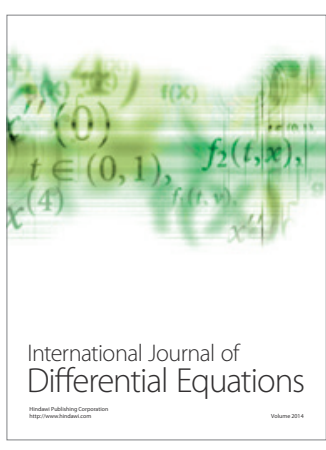
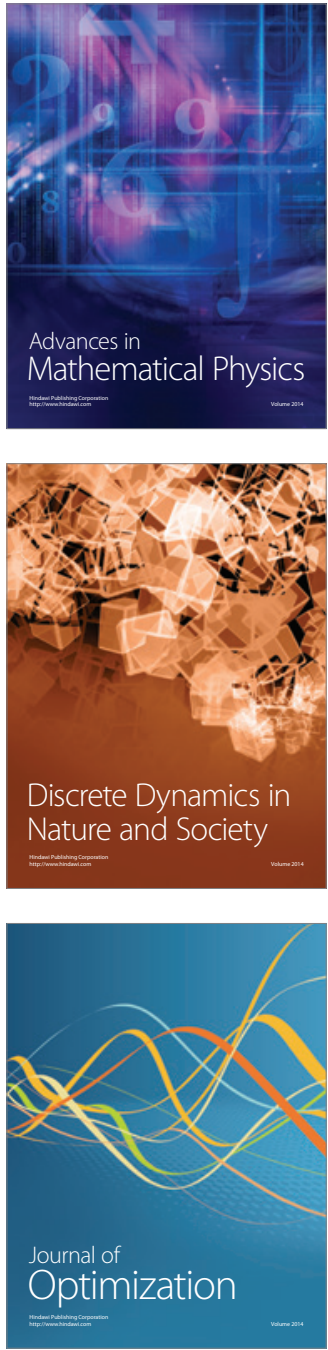